Article original

\title{
Etude de l'implication du polymorphisme fonctionnel Arg72Pro du gène TP53 dans la survenue du cancer colorectal et du carcinome Basocellu- laire dans la population de l'Ouest Algérien
}

\author{
Association of TP53 Arg72Pro Polymorphism with Risk of Colorectal cancer and basal \\ cell carcinoma in the Algerian Population
}

\begin{abstract}
Rym Abderrahmane ${ }^{\prime *}$, Lotfi Louhibi', Amina Boubekeur', Fatima Zohra Moghtit', Abdellah Boudjema', Meriem Abdi', Naima Meroufel', Fodil Mostefa', Nadhira Saidi-Mehtar'.

${ }^{1}$ Laboratoire de Génétique Moléculaire et Cellulaire, Département de Génétique Moléculaire Appliquée, Faculté des sciences de la nature et de la vie, Université des Sciences et de la Technologie d'Oran-Mohamed BOUDIAF-USTOMB-, BP 1505 El

M'naouer 31000, Oran, Algérie.
\end{abstract}

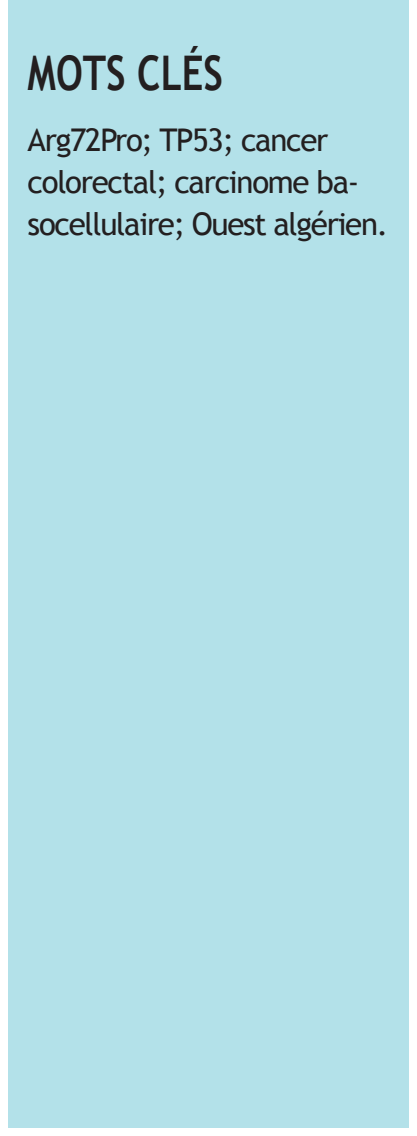

\section{Résumé}

Introduction - Le gène TP53 a fait l'objet de très nombreux travaux. Sa nature polymorphe et son rôle central dans la régulation du cycle cellulaire ont mis en évidence son potentiel de gène candidat dans la susceptibilité et la survenue de différents cancers. Bien que plusieurs polymorphismes du gène TP53 aient été étudiés comme facteurs de risque pour différents cancers, le plus largement étudié est le polymorphisme Arg72Pro (rs1042522), à l'origine d'une substitution d'une Arginie (Arg) en Proline (Pro) ou inversement, connu pour ses différentes fonctions biologiques.

Notre objectif est d'explorer la participation du polymorphisme (SNP) Arg72Pro du gène TP53, dans l'expressivité du cancer colorectal (CCR) et du carcinome basocellulaire $(C B C)$ dans une population de l'Ouest Algérien.

Matériels et Méthodes - Le déséquilibre de transmission allélique de ce SNP a été réalisé sur une population constituée de 116 individus atteints de CCR, 50 sujets atteints de CBC et enfin une population contrôle constituée de 121 individus sains, par la technique de Biologie Moléculaire PCR/RFLP (Polymerase Chain Reaction/ Restriction Fragment LengthPolymorphism).

Résultats - L'association du marqueur Arg72Pro avec le CBC a montré une augmentation significative de l'allèle Pro chez les cas par rapport aux contrôles ( $54 \%$ vs $46 \%$, $\mathrm{OR}=8,85$ [4,98-16,03], $\mathrm{p}<10-7)$. Cet allèle semble conférer un risque important de développer le CBC. Par ailleurs, les résultats n’ont montré aucune différence signi-

'Auteur correspondant: abderrahmane_rym@yahoo.fr 
ficative entre les sujets atteints de CCR et les contrôles, ce qui pourrait exclure son implication dans la survenue du CCR dans notre population ( $p>0.05$ ).

Conclusion - Le polymorphisme Arg72Pro du gène TP53 peut être considéré comme marqueur de risque pour le CBC mais pas pour le CCR.

\section{KEY WORDS}

Arg72Pro; TP53; colorectal cancer; basal cell carcinoma; West Algeria.

\section{Introduction}

Le gène TP53 est un gène suppresseur de tumeurs qui joue un rôle important dans l'arrêt du cycle cellulaire et l'apoptose. La perte de sa fonction cause à l'organisme la perte de son ultime défense contre les cellules aberrantes; ainsi les ADN lésés ne sont plus capables de stimuler l'auto destruction de celles-ci. Les cellules endommagées survivent et prolifèrent aboutissant ainsi à une progression vers l'état cancéreux [1].

Les modifications du gène suppresseur de tumeur TP53 sont les lésions génétiques les plus courantes dans les cancers humains. Il a été trouvé muté dans plus de $50 \%$ dans tous types de cancers confondus [2]. En plus des mutations, plusieurs polymorphismes dans le gène TP53 ont été détectés [3] et le polymorphisme Arg72Pro (rs1042522) est le plus largement étudié. Ce SNP est à l'origine d'une substitution d'une Arginie (Arg) en Proline (Pro) ou inversement, et est connu pour ses différentes fonctions biologiques [4]. En effet, différentes études ont montré que la forme p53 72Arg est plus efficace dans l'induction de l'apoptose, alors que la forme p53 72Pro a été suggérée meilleure pour induire un arrêt du cycle cellulaire en G1 et permettre ainsi la réparation de l'ADN.
Comme la voie P53 est une voie biologique hautement régulée, Il a été proposé que le SNP Arg72Pro (rs1042522) touchant le gène TP53, perturberait cette dernière en créant des changements des gènes transactivés par le facteur de transcription P53 [5]. Différentes études ont montré que la forme p53 72Arg est plus efficace dans l'induction de l'apoptose, tandis que la forme p53 72Pro a été suggérée pour induire un meilleur arrêt du cycle cellulaire et une réparation de l'ADN [6].

Il a été proposé donc que ces SNPs génèrent une variabilité interindividuelle dans les mécanismes contrôlant cette voie, d'où un risque variable de développer certaines maladies complexes telles que le cancer $[7,8]$.

Etant donné qu'il n'existe aucune donnée sur les polymorphismes Arg72Pro (rs1042522) du gène TP53 dans la population algérienne, nous avons entrepris une première étude permettant d'évaluer la fréquence de ce dernier dans un échantillon contrôle de la population. Les résultats obtenus serviront de référence pour les études cas/témoins concernant le carcinome basocellulaire et le cancer colorectal. Les résultats de cette étude permettraient de mettre en 
évidence une éventuelle association entre ce polymorphisme et la survenue du CCR et du CBC dans l'Ouest algérien. Par ailleurs, ils viendront confirmer ou infirmer ce qui a été rapporté dans les diverses publications.

\section{Matériels et méthodes}

\section{Matériel biologique}

Afin d'évaluer l'impact du gène suppresseur de tumeur TP53 sur le développement du CCR et du CBC, nous avons étudié le déséquilibre de transmission allélique des SNPs Arg72Pro du gène TP53 dans une population constituée de 116 individus atteints de CCR, 50 sujets atteints de CBC et enfin une population contrôle constituée de 121 individus sains.

- Population de sujets atteints du Cancer colorectal :

Elle est constituée de 116 malades; 70 hommes et 46 femmes; non apparentés, originaires de l'Ouest algérien et recrutés au niveau du service d'oncologie dirigé par le Pr Louafi « $\mathrm{CHU}$ d'ORAN ».

- Population de sujets atteints de Carcinome Basocellulaire :

Elle compte 50 patients de sexe masculin, âgés de 40 à 70 ans, non apparentés et originaires de l'Ouest algérien. Ces patients ont été recrutés au niveau du CHU d'ORAN.

- Population de témoins :

Elle comprend 121 individus ( 61 hommes et 60 femmes) originaires de l'Ouest algérien, et ne souffrant d'aucune pathologie au moment de l'étude. Les sujets dont le sang a été prélevé ont été soumis à un questionnaire retraçant les informations personnelles et les données utiles à notre étude. Celui-ci était accompagné d'un formulaire de consentement éclairé signé par toutes les personnes participant à cette étude. Toutes les précautions visant le respect de l'anonymat et la confidentialité des informations ont été rigoureusement respectées.

Tableau 1: Distribution des fréquences du polymorphisme Arg72Pro du gène TP53 chez les cas par rapport aux contrôles et son association avec le risque de survenue du CCR

\begin{tabular}{|l|c|c|c|c|}
\hline & $\begin{array}{c}\text { Contrôles } \\
(\mathrm{n}=121)\end{array}$ & $\begin{array}{c}\text { sujets CCR } \\
(\mathrm{n}=116)\end{array}$ & $\begin{array}{c}\text { OR } \\
(95 \% \mathrm{Cl})\end{array}$ & $\begin{array}{c}\mathrm{P} \\
\text { Value }\end{array}$ \\
\cline { 2 - 3 } & $\mathrm{N}(\%)$ & $\mathrm{N}(\%)$ & & \\
$\begin{array}{l}\text { Génotypes } \\
\text { Arg/Arg } \\
\text { Arg/Pro+Pro/Pro }\end{array}$ & $\begin{array}{c}102(84.29) \\
19(15.69)\end{array}$ & $\begin{array}{c}99(85.34) \\
17(14.65)\end{array}$ & $0.92(0.45-1.87)$ & 0.82 \\
\hline $\begin{array}{l}\text { Alleles } \\
\text { Arg } \\
\text { Pro }\end{array}$ & $\begin{array}{c}220(90.91) \\
22(9.09)\end{array}$ & $\begin{array}{c}209(90.09) \\
23(9.91)\end{array}$ & $1.1(0.6-2.03)$ & 0.75 \\
\hline
\end{tabular}

$N$ : effectif, \% : pourcentage, OR : odds ratio, $\mathrm{Cl}$ : intervalle de confiance, $p$ : significativité, a: génotype utilisé comme référence.

\section{Méthodes}

Le polymorphisme du codon 72 du gène TP53 a été exploré par la technique de Biologie Moléculaire PCR/RFLP (Polymerase Chain Reaction/Restriction Fragment LengthPolymorphism) pour explorer des ADN des populations témoin et de sujets atteints de CCR et CBC.

\section{- L'extraction d'ADN à partir du sang total}

Les ADN des différents sujets analysés pour le gène TP53 ont été extraits à partir du sang total selon la technique utilisant le $\mathrm{NaCl}[9]$.

\section{- PCR/RFLP}

La région qui encadre le codon 72 a été amplifié par PCR, en utilisant les amorces F:5'CGTTCTGGTAAGGACAAGGGTT3' et R: 5'TCCATGAGACTTCAATGCCTGG3'.

Un volume d'ADN génomique de 100ng est amplifié dans un mélange réactionnel de $25 \mu \mathrm{l}$ comprenant: $1 \mathrm{X}$ TP, $4 \mathrm{mM}$ de MgCL2, 0,2mM de d'NTP, 1 $\mu M$ d'amorces P1 et P2, et 0,25U de Taq polymérase (Ozyme, TAKARA). Les tubes ont été soumis à des cycles de température réitérés dans un thermocycleur qui suit le programme suivant: dénaturation à $94^{\circ} \mathrm{C}$ durant 5 minutes, 30 cycles composé de $94^{\circ} \mathrm{C}$ pendant 30 secondes, $60^{\circ} \mathrm{C}$ pendant 30 secondes, et $72^{\circ} \mathrm{C}$ pendant 30 seconde; et au final, l'étape d'extension à $72^{\circ} \mathrm{C}$ pendant 5 minutes.

Les amplimères obtenus ont été digérés par l'enzyme de restriction Btgl. Une quantité de $5 \mu$ des produits PCR est digérée par l'enzyme Btgl (Bacillus Thermoglucosidasius) (5U) dans un volume final de $15 \mu$ l en présence du tampon de l'enzyme TP10X $(1,5 \mu l$ ) et de $0,15 \mu$ le BSA (Bovine SerumAlbumin). La digestion est opérée toute la nuit (over night) dans un bain-marie à une température optimale de $37^{\circ} \mathrm{C}$.

-Analyses statistiques

Les fréquences de génotypage ont été vérifiées avec les attentes de Hardy-Weinberg par le test $x 2$. Le degré de significativité a été fixé à $p<0,05$. Le logiciel «Epilnfo TM version 7》 a été utilisé pour calculer les odd ratio (OR) avec leurs intervalles de confiance (IC) à $95 \%$.

\section{Résultats}

Analyse de la distribution allélique et génotypique du polymorphisme Arg72Pro du gène TP53 chez les sujets atteints de CCR :

L'analyse de la distribution des allèles Arg et Pro chez les sujets atteints de CCR par rapport aux témoins n'a montré aucune différence significative entre les deux groupes $(p>0,05)$. On retrouve l'allèle Pro et l'allèle Arg avec des fré 
Analyse de la distribution allélique et génotypique du polymorphisme Arg72Pro du gène TP53 chez les sujets atteints de $\mathrm{CBC}$ :

L'étude de l'association entre le marqueur Arg72Pro et le CBC a montré une augmentation significative de l'allèle Pro chez les malades par rapport aux contrôles ( $54 \%$ vs $46 \%$, $\mathrm{OR}=8,85$ [4,98-16,03], $\mathrm{p}<10-7)$. Cet allèle semble apporter un risque important à développer le $\mathrm{CBC}$ dans notre population (tableau 2).

Tableau 2. Distribution des fréquences alléliques et génotypiques du polymorphisme Arg72Pro du gène TP53 chez les CBC par rapport aux contrôles.

\begin{tabular}{|c|c|c|c|c|}
\hline & $\begin{array}{l}\text { Contrôles } \\
\mathrm{N}(\%)\end{array}$ & $\begin{array}{c}\text { Sujets CBC } \\
\text { N(\%) }\end{array}$ & $\begin{array}{c}\mathrm{OR} \\
(95 \% \mathrm{IC})\end{array}$ & $\begin{array}{c}P \\
\text { Value }\end{array}$ \\
\hline $\begin{array}{c}\text { Genotypes } \\
\text { Arg/Arg } \\
\text { Arg/Pro } \\
\text { Pro/Pro }\end{array}$ & $\begin{array}{c}75(79 \%) \\
16(17 \%) \\
3(3 \%)\end{array}$ & $\begin{array}{l}16(32 \%) \\
14(28 \%) \\
20(40 \%)\end{array}$ & $\begin{array}{c}1 \\
4,10(1,67-10,06) \\
31(7,62-175,84)\end{array}$ & $\begin{array}{c}0,001^{*} \\
0^{*}\end{array}$ \\
\hline $\begin{array}{l}\text { Alleles } \\
\text { Arg } \\
\text { Pro }\end{array}$ & $\begin{array}{c}166(88 \%) \\
22(12 \%)\end{array}$ & $\begin{array}{l}46(46 \%) \\
54(54 \%)\end{array}$ & $\begin{array}{c}1 \\
8,85(4,98-16,03)\end{array}$ & $0^{*}$ \\
\hline
\end{tabular}

$N$ : effectif, \%: pourcentage, OR: odds ratio, IC: intervalle de confiance, $p$ : significativité, a: génotype utilisé comme la catégorie de référence. "*: $p<0.05$ considéré comme statistiquement significatif.

Ces résultats sont confirmés au niveau des fréquences génotypiques car les génotypes comportant au moins un allèle Pro, présentent une association significative avec la maladie $(p<0,05)$.

\section{Discussion}

A notre grand avantage, notre étude est la première qui s'intéresse à évaluer l'association entre le CBC et le CCR avec le polymorphisme Arg72Pro sur un échantillon de la population de l'Ouest Algérien. Dans le même contexte, aucune étude similaire n'a été réalisée en Afrique d'où la difficulté de comparer nos résultats avec ceux de populations africaines, mais plutôt avec des populations caucasiennes et asiatiques.

Pour notre étude, les résultats rejettent l'association entre le polymorphisme Arg72Pro et le risque de développer le CCR. Ce résultat est en accord avec une méta analyse faite sur la population chinoise [10], et en désaccord avec plusieurs autres études, nous citerons comme exemple les populations grecques de race blanche et coréennes, qui ont rapporté respectivement les allèles Arg et Pro comme prédisposant au CCR $[11,12]$.

L'absence d'association cohérente du polymorphisme Arg72Pro avec le risque de cancer colorectal dans les différentes études peut être due à des différences de fréquences des allèles entre les groupes ethniques, à l'hétérogénéité génétique dans la pathogenèse du cancer colorectal, à différents facteurs environnementaux, et/ou à des restrictions de taille de l'échantillon. Il est également possible que ce polymorphisme soit en déséquilibre de liaison avec d'autres variantes étiologiques présumées qui seraient susceptibles de varier entre les différents groupes ethniques [13].

Pour le CBC, une association significative de l'allèle Pro du marqueur Arg72Pro a été constatée chez les cas par rapport aux contrôles. Ceci nous amène à dire qu'il pourrait être considéré comme un allèle prédisposant pour cette pathologie.

Ces résultats sont similaires à ceux retrouvés dans une étude cas-témoins, réalisée au sud-est de Taiwan, qui a mis en évidence une augmentation de l'incidence du CBC chez les personnes porteuses du génotype Pro/Pro, comparées aux génotypes Pro/Arg et Arg/Arg [14]. Par contre, dans une étude cas-témoins menée par Han et al, en 2006, un risque accru de développer le CBC a été associé avec l'allèle Arg72 du gène TP53 (OR=1.79 [1,1 à 3,17]) [15]. De même pour l'équipe de McGregor et al, qui a trouvé une association entre l'allèle Arg et le CBC [16].

Enfin, pour les autres études, aucune association n'a été retrouvée entre le polymorphisme Arg72Pro du gène TP53 et le $C B C[17,18,19,20,21,22]$. Pareillement pour une méta analyse menée par De-Ke Jiang et al, où aucune association significative n'a été constatée entre le SNP Arg72Pro et différents types de cancer de peau, y compris le CBC [23].

Toutefois, l'importance du polymorphisme du codon-72 réside dans le fait que ce dernier est situé dans un domaine riche en proline, hautement conservé, et impliqué dans la liaison à l'ADN. Plusieurs études ont montré des différences claires et fonctionnelles entre les deux isoformes. En effet, l'expression de TP53-72Arg dans les cellules est liée à une induction plus renforcée de l'apoptose [23]. En effet, il a été démontré que l'allèle Arg se lie plus spécifiquement à la PIN1 de Proly-isomérase, entraînant ainsi une augmentation de l'acétylation de P53 ce qui conduirait à sa dissociation de l'inhibiteur de l'apoptose laspp, par rapport à l'allèle Pro [24]. Le potentiel apoptotique spécifique au variant TP53$72 \mathrm{Arg}$ a été récemment confirmé dans des modèles de souris $[25,26]$. En outre, des cultures cellulaires de fibroblastes dermiques provenant de souris ont montré que le variant Tp53-72Pro avait une meilleure capacité à induire l'arrêt du cycle cellulaire et la sénescence que l'allèle Arg en raison de sa capacité accrue à transactiver p21 / WAF1 [27]. D'autres part, les cellules exprimant le variant Tp53-72Pro sont également plus efficaces pour l'activation spécifique de plusieurs gènes cibles de réparation de l'ADN et sont associées à une plus grande capacité de réparation de l'ADN que 
les cellules exprimant l'allèle TP53-72Arg [28].

Le consensus actuel est que Tp53-72Arg est plus efficace pour induire l'apoptose et la protection des cellules du développement néoplasique. Par ailleurs, le variant TP53-72Pro est plus efficace pour induire l'arrêt du cycle cellulaire et la sénescence [29]. L'élucidation de l'effet des polymorphismes du gène TP53 sur le risque de cancer est un défi qui attire l'attention ces dernières années. A ce jour, aucune conclusion ne peut être tirée sur les rôles des polymorphismes et des mutations du gène TP53. En outre, d'importantes études sur les différentes ethnies, enquêtent sur un plus large éventail sur les variantes du gène TP53 afin de les inclure dans l'approche haplotype-tagging (marquage via les haplotypes) [30]. Malgré des progrès impressionnants dans la compréhension des mécanismes de la structure et de la fonction P53, la recherche n'a pas encore généré des applications de large impact sur la gestion et le traitement du cancer. II est peu probable que les altérations du gène TP53 puissent être cliniquement utiles, et utilisées comme marqueur de pronostic de routine pour le CCR et le CBC.

\section{Conclusion}

L'analyse du polymorphisme TP53 Arg72Pro dans la population algérienne, suggère que ce polymorphisme ne pourrait pas constituer un facteur de risque pour la survenue du CCR. Par contre, il semble constituer un risque important de développer le CBC.

\section{Remerciements}

Nous tenons à remercier l'ensemble des patients qui ont bien voulu participer à notre étude ainsi que tout le personnel hospitalier qui nous ont permis de récolter notre échantillonnage dans les meilleures conditions.

\section{Conflits d'intérêt}

Il n'y a aucun conflit d'intérêt entre les auteurs.

\section{Références bibliographiques}

[1] Whibley C, Pharoah PD, Hollstein M. p53 polymorphisms: Cancer implications. Nat Rev Cancer. 2009;9:95-107.

[2] Sherr, C. J. «Principles of tumor suppression.» Cell 2004. 116(2): 235-246.

[3] Green, D. R. and G. Kroemer (2009). "Cytoplasmic functions of the tumour suppressor p53.» Nature 458(7242): 1127-1130.

[4] Matlashewski GJ, Tuck S, Pim D, Lamb P, Schneider J, Crawford LV. Primary structure polymorphism at amino acid residue 72 of human p53. Mol Cell Biol. 1987;7:961-3.
[5] Moreau F, Matlashewski G. Molecular analysis of different allelic variants of wild-type human p53. Biochem Cell Biol. 1992;70:1014-9.

[6] Thomas M, Kalita A, Labrecque S, Pim D, Banks L, Matlashewski G. Two polymorphic variants of wild-type p53 differ biochemically and biologically. Mol Cell Biol. 1999;19:1092-100.

[7] Post SM, Quintás-Cardama A, Pant V, Iwakuma T, Hamir A, Jackson JG, et al. A high-frequency regulatory polymorphism in the p53 pathway accelerates tumor development. Cancer Cell. 2010;18:22030.

[8] Whibley, C., P. D. Pharoah, et al. (2009). «p53 polymorphisms: cancer implications.» Nat Rev Cancer 9(2): 95-107.

[9] Miller, S. A., D. D. Dykes, et al. (1988). «A simple salting out procedure for extracting DNA from human nucleated cells.» Nucleic Acids Res 16(3): 1215.

[10] Zhu ZZ, Wang AZ, Jia HR, Jin XX, He XL, Hou LF, Zhu G (2007) Association of the TP53 codon 72 polymorphism with colorectal cancer in a Chinese population. Jpn J ClinOncol 37(5):385-390

[11] Dakouras A, Nikiteas N, Papadakis E, Perakis M, Valis D, Rallis G, Tzanakis N, Peros G, Tsigkris C, Kittas C, Karakitsos P. P53Arg72 homozygosity and its increased incidence in left-sided sporadic colorectal adenocarcinomas, in a Greek- Caucasian population. Anticancer Res 28 2008; (2A):1039-1043.

[12] Cao Z, Song JH, Park YK, Maeng EJ, Nam SW, Lee JY, Park WS. The p53 codon 72 polymorphism and susceptibility to colorectal cancer in Korean patients. Neoplasma 2009; 56 (2):114-118.

[13] Sjalander A, Birgander R, Athlin L, Stenling R, Rutegard J, Beckman L, Beckman G. P53 germ line haplotypes associated with increased risk for colorectal cancer. Carcinogenesis 1995;16:1461-1464.

[14] Almquist LM, Karagas MR, Christensen BC, Welsh MM, Perry AE, Storm CA, et al. The role of TP53 and MDM2 polymorphisms in TP53 mutagenesis and risk of non-melanoma skin cancer. Carcinogenesis. 2011;32:327-30;

[15] Han J, Cox DG, Colditz GA, Hunter DJ. La p53 codon 72 polymorphisme, les coups de soleil, et le risque de cancer de la peau dans les femmes américaines de race blanche. MolCarcinog. 2006; 45: 694-700.

[16] McGregor JM, Harwood CA, Brooks L, Fisher SA, Kelly DA, O'nions J, et al. Relationship between p53 codon 72 polymorphism and susceptibility to sunburn and skin cancer. J Invest Dermatol. 2002;119:84-90;

[17] Pezeshki A, Sari-Aslani F, Ghaderi A, Doroudchi M. p53 codon 72 polymorphism in basal cell carcinoma of the skin. PatholOncol Res. 2006;12:29-33.

[18] Rizzato C, Scherer D, Rudnai P, Gurzau E, Koppova K, Hemminki $\mathrm{K}$, et al. POMC and TP53 genetic variability and risk of basal cell carcinoma of skin: Interaction between host and genetic factors. J Dermatol Sci. 2011;63:47-54.

[19] Dokianakis DN, Koumantaki E, Billiri K, Spandidos DA. P53 codon 72 polymorphism as a risk factor in the development of HPV-associated non-melanoma skin cancers in immunocompetent hosts. Int $\mathrm{J}$ 
Mol Med. 2000;5:405-9.

[20] Bendesky A, Rosales A, Salazar AM, Sordo M, Peniche J, Ostrosky-Wegman P. p53 codon 72 polymorphism, DNA damage and repair, and risk of non-melanoma skin cancer. Mutat Res. 2007;619:3844.

[21] Bastiaens MT, Struyk L, Tjong-A-Hung SP, Gruis N, terHuurne $\mathrm{J}$, Westendorp RG, et al. Cutaneous squamous cell carcinoma and p53 codon 72 polymorphism: A need for screening? MolCarcinog. 2001;30:56-61.

[22] Dumont P, Leu JI, Della Pietra AC, 3rd, George DL, Murphy M. The codon 72 polymorphic variants of $p 53$ have markedly different apoptotic potential. Nature genetics. 2003;33:357-365.

[23] Yanli Tian, Li Li, and Rongya Yang; p53 Arg72Pro polymorphism and risk of basal cell carcinoma: a meta analysis; Int J Clin Exp Med. 2015; 8(2): 2350-2356

[24]Mantovani F, Tocco F, Girardini J, Smith P, Gasco M, et al. The prolylisomerase Pin1 orchestrates p53 acetylation and dissociation from the apoptosis inhibitor iASPP. Nature structural \& molecular biology. 2007;14:912-920.
[25] Zhu F, Dollé MET, Berton TR, Kuiper RV, Capps C, et al. Mouse models for the p53 R72P polymorphism mimic human phenotypes. Cancer Res. 2010;70:5851-5859. [PMC free article

[26] Azzam GA, Frank AK, Hollstein M, Murphy ME. Tissue-specific apoptotic effects of the p53 codon 72 polymorphism in a mouse model. Cell Cycle. 2011;10:1352-1355.

[27] Frank AK, Leu JI, Zhou Y, Devarajan K, Nedelko T, et al.The codon 72 polymorphism of p53 regulates interaction with NF-\{kappa\}B and transactivation of genes involved in immunity and inflammation. Molecular and cellular biology. 2011;31:1201-1213.

[28] Siddique M, Sabapathy K. Trp53-dependent DNA-repair is affected by the codon 72 polymorphism. Oncogene. 2006;25:3489-3500.

[29] Matlashewski GJ, Tuck S, Pim D, Lamb P, Schneider J, Crawford LV (1987) Primary structure polymorphism at amino acid residue 72 of human p53. Mol Cell Biol 7(2):961-963.

[30] Vazquez A1, Bond EE, Levine AJ, Bond GL.The genetics of the p53 pathway, apoptosis and cancer therapy. Nat Rev Drug Discov. 2008 Dec;7(12):979-87. 